\title{
Local control after stereotactic radiosurgery for brain metastases in patients with melanoma with and without $B R A F$ mutation and treatment
}

\author{
David Ly, MD, ${ }^{1}$ Hilary P. Bagshaw, MD, ${ }^{1}$ Christopher J. Anker, MD, ${ }^{2}$ Jonathan D. Tward, MD, PhD, ${ }^{1}$ \\ Kenneth F. Grossmann, MD, PhD, ${ }^{3}$ Randy L. Jensen, MD, PhD, ${ }^{4}$ and Dennis C. Shrieve, MD, PhD ${ }^{1}$ \\ 1Department of Radiation Oncology and ${ }^{3}$ Division of Oncology, Huntsman Cancer Hospital, and ${ }^{4}$ Department of Neurosurgery, \\ University of Utah, Salt Lake City, Utah; and 2Division of Radiation Oncology, University of Vermont Cancer Center, \\ Burlington, Vermont
}

OBJECT BRAF inhibitors improve progression-free and overall survival in patients with metastatic melanoma. Brain
metastases are common, and stereotactic radiosurgery (SRS) has been used, resulting in excellent local control.
Because BRAF inhibitors are associated with intracranial responses, the authors hypothesized that BRAF inhibitors
would improve local control in patients with melanoma who are receiving SRS for brain metastases. METHODS The authors retrospectively identified patients with metastatic melanoma who had been tested for BRAF mutation and treated with SRS for brain metastases. Patients with previous resection, multiple brain metastases, or multiple courses of SRS were eligible. SRS was delivered in a single fraction to a median dose of $2000 \mathrm{cGy}$. Patients with a $B R A F$ mutation were treated with a BRAF inhibitor on the basis of physician preference.

RESULTS The authors identified 52 patients who were treated in 82 treatment sessions for 185 brain metastases and 13 tumor beds. At a median follow-up of 10.5 months, the 1 -year local control rate was $69.2 \%$. At 1 year, the local control rate for brain metastases in patients with $B R A F$ mutation with BRAF treatment was $85.0 \%$, and the local control rate for brain metastases in those without BRAF treatment was $51.5 \%(p=0.0077)$. The rates of distant brain failure, freedom from whole-brain radiation, and overall survival were not different on the basis of BRAF mutation status or inhibitor therapy. The number of new intratumoral hemorrhages after SRS was increased significantly in patients with BRAF treatment. CONCLUSIONS Treatment with BRAF inhibitors was associated with improved local control after SRS in patients with melanoma and brain metastases. An increased number of intratumoral hemorrhages was associated with BRAF inhibitor therapy.

http://thejns.org/doi/abs/10.3171/2014.9.JNS141425

KEY WORDS stereotactic radiosurgery; melanoma; BRAF; brain metastases; BRAF inhibitor

$\mathrm{I}$ $\mathrm{N}$ recent years, the incidence of melanoma in all age groups has been increasing in the United States..$^{20}$ The cumulative incidence of brain metastases for all patients with melanoma is between $7.4 \%$ and $11.1 \% .16,35,37,42$ Stereotactic radiosurgery (SRS) is an effective treatment option that results in high rates of local control. ${ }^{24,32}$ SRS treatment of brain metastases in select patients with melanoma may be associated with improved survival. ${ }^{24,30}$

Systemic management of metastatic melanoma has focused on agents that can improve overall survival and intracranial response. BRAF is a protein kinase that has been implicated in cell proliferation and differentiation and is mutated in $70 \%$ of patients with melanoma. ${ }^{8}$ Mutation in the $B R A F$ gene protects cells from apoptosis. ${ }^{22}$ Targeted therapies for the $B R A F \mathrm{~V} 600 \mathrm{E}$ mutation have been developed. Vemurafenib was shown in a randomized controlled triall $^{7}$ to prolong overall survival. In addition, dabrafenib has been shown to improve progression-free survival. ${ }^{17}$ The use of BRAF inhibitors was studied in patients with melanoma brain metastases after case reports showed favorable responses. ${ }^{11,39}$ Dabrafenib showed an overall intracranial response of $39 \%$ in patients with melanoma if they did not have previous local treatment. ${ }^{25}$ Vemurafenib was studied in patients with untreated melanoma brain metastases and was associated with an intracranial response of $53 \% .^{9}$ 
Although systemic agents have shown promise, local therapies such as surgery and irradiation remain the mainstay of treatment for brain metastases. In a study of vemurafenib, patients were eligible only if their disease had progressed despite local therapy. In the study, $25 \%$ of the 24 subjects underwent SRS. ${ }^{9}$ Because of the favorable local control rates provided by SRS and the promise of BRAF inhibitors in the extracranial/intracranial setting, it is likely that many patients with melanoma and brain metastases will undergo both treatments.

We hypothesize that the addition of BRAF inhibitor therapy will improve local control in patients with melanoma who undergo SRS for brain metastases.

\section{Methods \\ Patient Selection}

We retrospectively identified patients who were 18 years or older with metastatic melanoma who received their first course of radiation for brain metastases with SRS at the University of Utah and completed testing for $B R A F$ mutation between 2009 and 2012. This study was approved by the University of Utah institutional review board, which waived the need for informed consent. Patients who had resection, multiple brain metastases, or multiple courses of SRS were included in this study. Patients with previous brain irradiation were excluded.

\section{Radiation Therapy}

SRS was delivered using a frameless linear accelerator-based radiosurgery unit. Target volumes were treated in a single treatment fraction by using a dynamic conformal arc technique with 6-MV photon beams. Stereotactic treatment planning was completed using the iPlan system (BrainLAB). The planned target volume was the brain metastasis or resected tumor bed without additional expansion. The prescription isodose surface was selected such that $\geq 95 \%$ of the target volume was encompassed by the prescription isodose volume and $\geq 99 \%$ of the target volume was covered by $\geq 95 \%$ of the prescription dose. ${ }^{18}$ The median dose delivered was 2000 cGy. Salvage treatment for recurrent lesions included SRS and surgery in select cases. Salvage treatment for distant brain disease was based on physician preference and included SRS or wholebrain radiation therapy. Whole-brain radiation therapy was administered using opposed laterals with 6-MV photon beams to a median dose of $3750 \mathrm{cGy}$ in 15 fractions.

\section{BRAF Mutation Testing and Inhibitor Treatment}

Patients with metastatic melanoma had their tissue tested for $B R A F$ mutation V600E at the University of Utah between 2009 and 2012. Patients who did not have the $B R A F$ V600E mutation (wild-type $B R A F$ ) were not treated with BRAF inhibitor therapy. Patients with the $B R A F$ V600E mutation were treated with a BRAF inhibitor (dabrafenib or vemurafenib) on the basis of physician preference, but it was typically initiated after other systemic treatments had failed (median 2 systemic treatments, range $0-5$ systemic treatments). Patients were treated with a BRAF inhibitor for $\geq 60$ days. BRAF inhibitor therapy could be initiated before or after SRS. The majority of the treated lesions $(53.9 \%)$ were not treated with a BRAF inhibitor within a 30-day period before or after SRS. If the patient was on a BRAF inhibitor or was about to start a BRAF inhibitor (46.1\%), a washout period was initiated before and after SRS (median 7 days, range 1-20 days). Of the 17 patients treated with BRAF inhibitor therapy, 9 were treated with $150 \mathrm{mg}$ of oral dabrafenib twice daily, 4 were treated with $720 \mathrm{mg}$ of oral vemurafenib twice daily, 3 were treated with $960 \mathrm{mg}$ of oral vemurafenib twice daily, and $1 \mathrm{had}$ unknown BRAF inhibitor treatment.

\section{Definition of End Points}

Patients were followed in multidisciplinary brain tumor clinics with serial contrast-enhanced brain MRI. The primary end point was local control; treatment failure of a brain metastasis was defined as retreatment with surgery and/or radiotherapy and/or an increase in the ratio of the axial, sagittal, or coronal area as noted by the product of the length and width in the given plane by $25 \%$ on subsequent T1-weighted post-contrast-enhanced MR images after SRS. Local control was timed from the date of SRS. ${ }^{21}$ Patients were censored if they were lost to followup, developed a hemorrhage of a treated lesion (as seen on a T1-weighted image), or at the start of any subsequent whole-brain radiation therapy secondary to distant brain disease. Distant brain failure was defined as development of new brain metastases on the T1 post-contrast-enhanced MR images obtained after the date of the SRS. Patients were censored for distant brain failure if they were lost to follow-up. Overall survival was calculated at the time of death from any cause from the time of SRS. Patients were censored for overall survival if they were lost to followup. Hemorrhage was identified as a hyperintense signal on the T1-weighted MR images before SRS and on all subsequent MRI scans.

\section{Statistical Analysis}

Statistical analysis was completed using StatsDirect 2.7.9 statistical software. The Student t-test (unpaired, 2 -sided test) for the comparison of means and the chisquare test for comparisons of proportions were used to assess differences in patient demographics. The chisquare test and the Fisher exact test were used for univariate analyses to examine the association of the end point and given outcome. We used the Kaplan-Meier method to assess the time to the end point. To compare the survival distributions of 2 samples, the log-rank test was used. A p value of $\leq 0.05$ was considered significant.

\section{Results \\ Demographics}

We identified 52 patients (median follow-up time 10.5 months) who met the study criteria. A total of 198 brain lesions (185 brain metastases and 13 operative beds) were treated in 82 treatment sessions (median 1 session, range 1-6 sessions) with a median dose of $2000 \mathrm{cGy}$ (range 1500-2400 cGy). Complete demographic data are shown in Table 1. Of the 52 patients who were treated, $31 \mathrm{had}$ the $B R A F$ mutation and 21 had the wild-type $B R A F$ gene. Of the patients who were positive for $B R A F$ mutation, 17 (54.8\%) were treated with a BRAF inhibitor. Of the 198 lesions treated with SRS, 21 lesions were treated with 
TABLE 1. Patient demographics

\begin{tabular}{lc}
\hline \multicolumn{1}{c}{ Demographic } & Patient Data \\
\hline Male (no. [\%]) & $40(77)$ \\
\hline Female (no. [\%]) & $12(23)$ \\
\hline Age (median [range]) (yrs) & $52(19-64)$ \\
\hline Treatment vol (median [range]) $\left(\mathrm{cm}^{3}\right)$ & $0.34(0.01-29.11)$ \\
\hline Dose (median [range]) (cGy) & $2000(1500-2400)$ \\
\hline Wild-type BRAF (no. [\%]) & $21(40.4)$ \\
\hline Mutated BRAF (no. [\%]) & $31(59.6)$ \\
\hline BRAF treatment (no. [\%]) & $17(54.8)$ \\
\hline Dabrafenib & $9(53)$ \\
\hline Vemurafenib & $7(41)$ \\
\hline Unknown & $1(5.9)$ \\
\hline KPS score (median [range]) & $90(50-100)$ \\
\hline GPA (median [range]) & $4(2-4)$ \\
\hline Extracranial disease present (no. [\%]) & $44(84.6)$ \\
\hline Previous systemic agents (median) & 2 \\
\hline GPA = Graded Prognostic Assessment: KPS = Karnofsky Performance Scale.
\end{tabular}

BRAF therapy before SRS, 34 lesions were treated with BRAF therapy before and after SRS, 41 lesions were treated with BRAF therapy after SRS, and 102 lesions were not treated with BRAF therapy. There were no significant differences, other than mutation status, between the patients treated with or without a BRAF inhibitor (Table 2).

\section{Local Control}

The 1-year local control rate for all the brain lesions was $69.2 \%$ (Fig. 1). There were a total of 38 treatment failures; 14 of 59 (23.7\%) failures occurred in the lesions of patients with wild-type $B R A F$, and 24 of $139(17.3 \%)$ failures occurred in the lesions of patients with $B R A F$ mutation. Of the 185 patients with brain metastases, 41 (22.2\%) had a complete response. At 1 year, the local control rate for brain lesions in patients with wild-type $B R A F$ was $67.1 \%$, whereas the 1-year local control rate for brain lesions in patients with $B R A F$ mutation was $70.0 \%$ ( $\mathrm{p}=$ 0.12). In patients with $B R A F$ mutation, BRAF inhibitor treatment was associated with improved local control (Fig. 2) $(\mathrm{p}=0.0077)$. At 1 year, in patients with $B R A F$ mutation, the local control rate for brain lesions for those who had BRAF inhibitor treatment was $85.0 \%$, and the local control rate for those who did not have BRAF inhibitor treatment was $51.5 \%$. The significance level for local control did not change when the patients with wild-type $B R A F$ were added to the cohort (Fig. 3). At 1 year, the local control rate for brain lesions in patients who had BRAF inhibitor treatment was $85.0 \%$, whereas the local control rate for patients without BRAF inhibitor treatment was $60.0 \%$ ( $\mathrm{p}=0.013)$. However, there was no difference in local control when we compared the patients with $B R A F$ mutation who did not have BRAF inhibitor treatment (hazard ratio $[\mathrm{HR}] 2.44,95 \%$ confidence interval $[\mathrm{CI}] 1.13-5.32)$ and the patients with wild-type $B R A F$ (HR $0.99,95 \%$ CI $0.43-2.31)$. We also evaluated the impact of BRAF inhibitor therapy before SRS versus no BRAF inhibitor therapy and found no association with local control when BRAF
TABLE 2. Demographics according to BRAF treatment

\begin{tabular}{|c|c|c|c|}
\hline \multirow[b]{2}{*}{ Demographic } & \multicolumn{2}{|c|}{ BRAF Inhibitor Treatment } & \multirow[b]{2}{*}{$\mathrm{p}$ Value } \\
\hline & No $(n=35)$ & Yes $(n=17)$ & \\
\hline Mean age (yrs) & 58.8 & 50.1 & 0.051 \\
\hline Male (no. [\%]) & $27(77.1)$ & $13(76.5)$ & 0.96 \\
\hline Female (no. [\%]) & $8(22.9)$ & $4(23.5)$ & \\
\hline Median KPS score & 90 & 90 & 0.58 \\
\hline GPA (median [range]) & $4(2-4)$ & $4(2-4)$ & 0.38 \\
\hline BRAF mutation (no. [\%]) & $14(40)$ & $17(100)$ & $<0.0001$ \\
\hline Extracranial disease (no. [\%]) & $28(80)$ & $16(94)$ & 0.19 \\
\hline $\begin{array}{l}\text { No. of sites of extracranial } \\
\text { disease (median [range]) }\end{array}$ & $3(0-5)$ & $2(0-5)$ & 0.77 \\
\hline $\begin{array}{l}\text { No. of systemic agents before } \\
\text { BRAF inhibitor treatment } \\
\text { (median [range]) }\end{array}$ & $2(0-5)$ & $2(0-4)$ & 0.72 \\
\hline
\end{tabular}

inhibitor therapy was initiated before SRS (HR 1.16, 95\% CI $0.47-2.86 ; \mathrm{p}=0.75$ ).

\section{Distant Brain Failure}

The 1-year distant brain control rate was $33.0 \%$ (median time to failure 5.5 months). At 1 year, the distant brain control rates in patients with wild-type $B R A F$ and those with $B R A F$ mutation were $33.0 \%$ and $32.3 \%$, respectively $(\mathrm{p}=$ 0.25 ); there was no association with $B R A F$ mutation status and distant brain control. In patients with $B R A F$ mutation, BRAF inhibitor treatment was not associated with distant brain failure $(\mathrm{p}=0.97)$. Similarly, distant brain failure was not affected when patients with wild-type $B R A F$ who did not have BRAF inhibitor treatment were included in the cohort compared with patients with $B R A F$ mutation who had BRAF inhibitor treatment $(\mathrm{p}=0.54)$.

\section{Freedom From Whole-Brain Radiation Therapy}

Patients in this series who experienced distant brain failure were treated with additional courses of SRS or whole-brain radiation therapy. There were 30 additional SRS courses. Whole-brain radiation was administered to $17(33.0 \%)$ of the 52 patients who were treated for brain metastases. The rate of 1-year freedom from whole-brain radiation therapy was $62.0 \%$ (median time to whole-brain

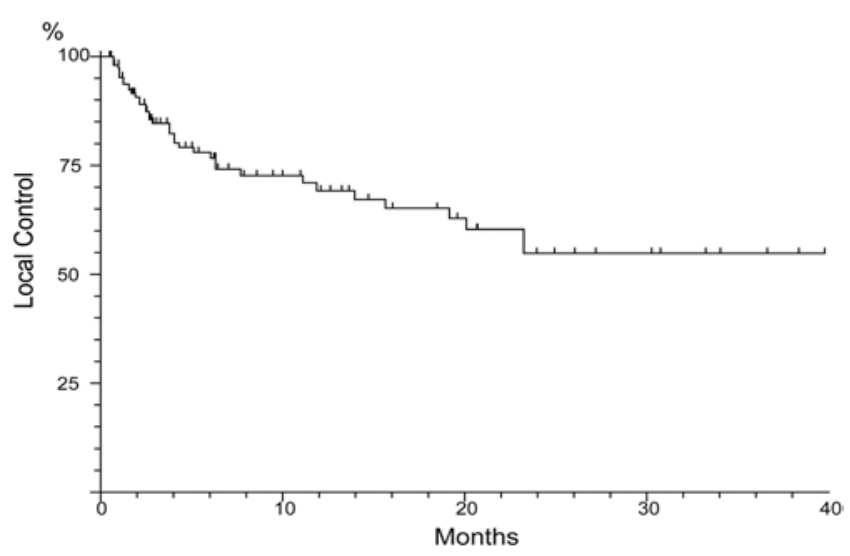

FIG. 1. Local control rates for all patients. 


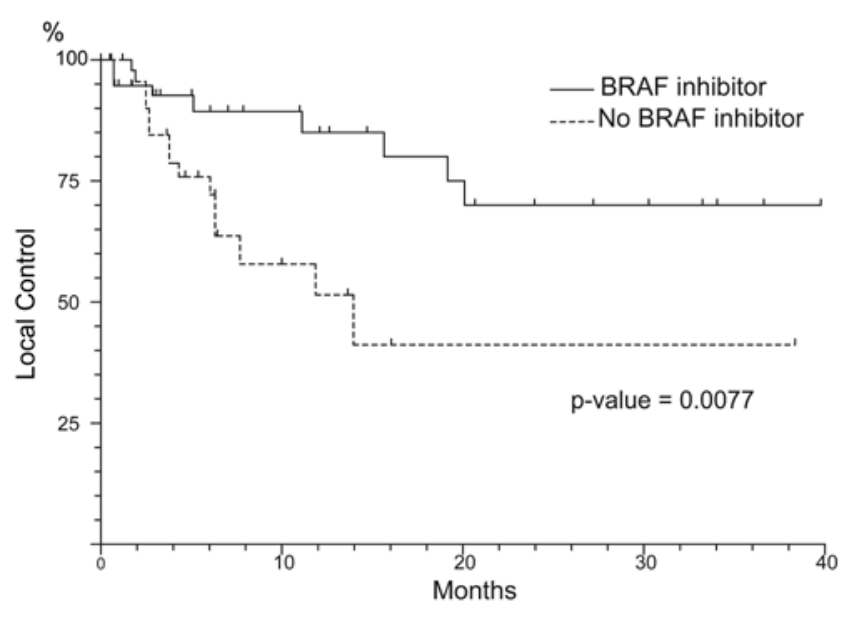

FIG. 2. Improved local control rates in patients with BRAF mutation who are treated with a BRAF inhibitor.

irradiation 23.6 months). The use of BRAF inhibitor treatment did not decrease the use of whole-brain radiation ( $\mathrm{p}$ $=0.46$ ).

\section{Overall Survival}

Of 52 subjects, $36(69.2 \%)$ had died by the last followup. At 1 year, the overall survival rate was $48.0 \%$ (median survival 12 months). Survival was not associated with $B R A F$ mutation status; at 1 year, the survival rates for patients with $B R A F$ mutation and those with wild-type $B R A F$ were $50.0 \%$ and $45.0 \%$, respectively $(\mathrm{p}=0.30)$. BRAF inhibitor treatment in patients who had $B R A F$ mutation was not associated with overall survival. At 1 year, the overall survival rates were $50.2 \%$ and $42.9 \%$ for patients who had BRAF inhibitor treatment and those who had no treatment, respectively $(\mathrm{p}=0.82)$. Overall survival did not change when the patients with wild-type $B R A F$ were included in the analysis $(\mathrm{p}=0.90)$. Of the 36 deaths, 6 were attributed to unknown causes, 2 were attributed to infectious etiologies, and 8 were attributed to systemic disease. Twenty (55.6\%) of the 36 deaths were related to CNS etiology (metastases, hemorrhage, etc.). Of the 20 deaths attributed to CNS causes, 15 (75.0\%) were related to intratumoral hemorrhage.

\section{Hemorrhage}

Of the 198 treated lesions, 27 (13.6\%) were associated with intratumoral hemorrhage before SRS. Hemorrhage before treatment did not differ by $B R A F$ mutation status $(\mathrm{p}=0.86)$; of the 50 lesions in patients with wild-type $B R A F, 8$ hemorrhaged before treatment $(16 \%)$, whereas of the 127 lesions in patients with $B R A F$ mutation, 19 hemorrhaged before treatment (15\%). Intratumoral hemorrhage developed in 50 lesions (29.2\%) after SRS. The median time to a new hemorrhage was 49 days from SRS. New intratumoral hemorrhage after SRS was not associated with $B R A F$ status $(\mathrm{p}=0.42)$. In addition, we did not find an association with hemorrhage and the treated volume of the lesion (HR 1.02, 95\% CI 0.99-1.06; p = 0.17). Increased hemorrhage risk was associated with BRAF inhibitor treatment in patients with $B R A F$ mutation; in patients who were treated with a BRAF inhibitor and in those who were

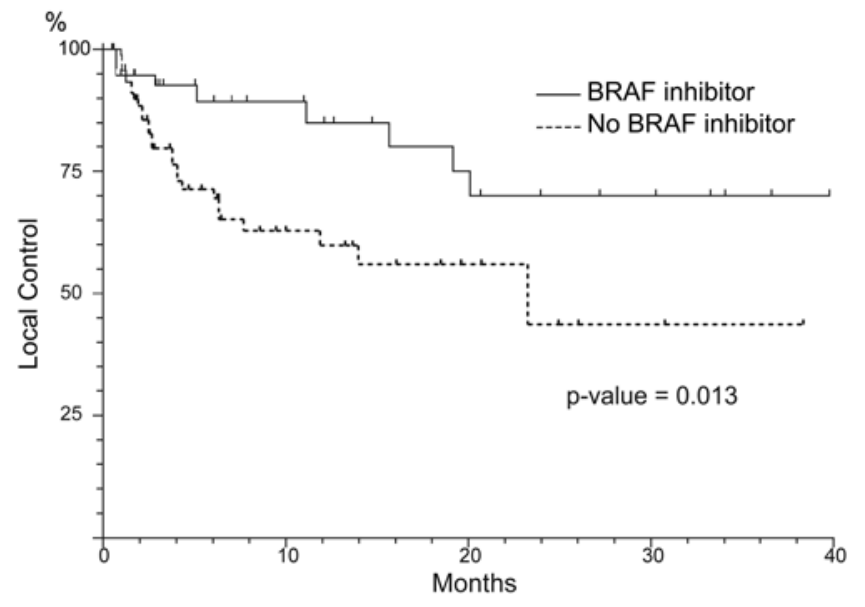

FIG. 3. Improved local control rates in patients who are treated with a BRAF inhibitor.

not, the 1-year rates of freedom from intratumoral hemorrhage were $39.3 \%$ and $77.0 \%$, respectively (Fig. 4) $(\mathrm{p}=$ $0.0003)$.

\section{Univariate Analysis}

Univariate analysis was completed to assess the presence of associations between patient/treatment characteristics and local control of the treated volume. $B R A F$ mutation status, treatment volume, age, sex, radiation dose, and whether the patient had surgery before SRS were not associated with local control (Table 3). Because no other variables were significant for local control, a multivariate analysis was not completed.

\section{Discussion}

The prognosis for patients with metastatic melanoma has improved with newer systemic agents, including BRAF inhibitors. ${ }^{7}$ Brain metastases in this patient population continue to be a problem. The treatment of brain metastases requires a multimodal approach. SRS is an effective tool for controlling brain metastases in patients with melanoma; our study shows a local control rate of $69.2 \%$ at 1 year. This rate is consistent with those from previous studies, which have estimated 1-year local control rates after SRS of between $49 \%$ and $94.3 \% .4,19,24,26,30,32,38$

In our review, patients with melanoma who underwent SRS for brain metastases and BRAF inhibitor therapy had improved local control. At 1 year, the local control rates in patients with $B R A F$ mutation who had BRAF treatment and those who did not have BRAF treatment were $85.0 \%$ and $51.5 \%$, respectively. Previous studies of BRAF inhibitors in the intracranial setting assessed the overall response to BRAF inhibitor therapy, but they did not evaluate local control.9,25 Twelve patients who received both radiation and vemurafenib demonstrated a 6-month local control rate of $75 \% .{ }^{29}$

The use of BRAF inhibitors and radiation therapy may have a synergistic effect. Because of the reported dermal reactions from radiation therapy and BRAF inhibitor therapy, BRAF inhibitors may cause radiosensitization. ${ }^{2}$ It was noted that, in a melanoma cell line, the addition of targeted agents to inhibit BRAF can enhance radiosensitization. ${ }^{34}$ 


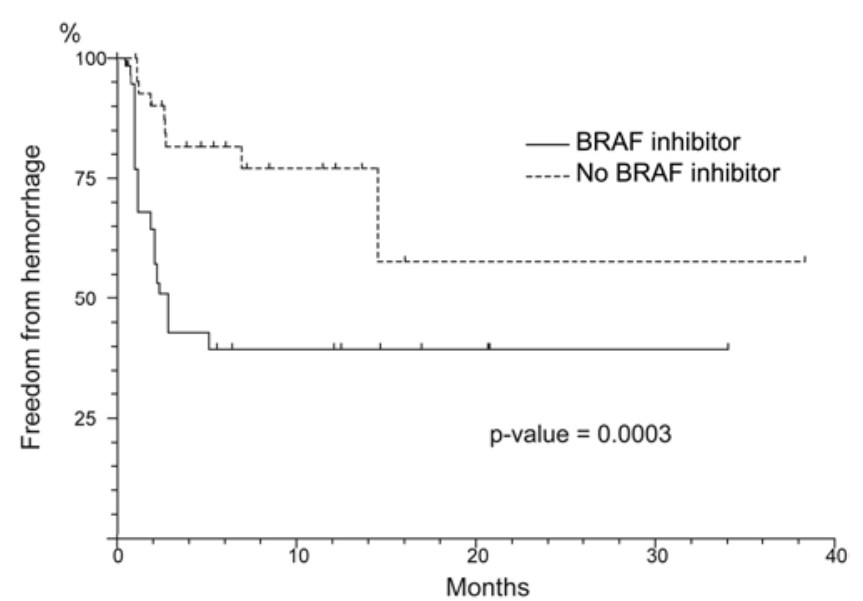

FIG. 4. BRAF inhibitor treatment is associated with the development of hemorrhage.

Radiation therapy has been shown to increase permeability in the blood-brain barrier. ${ }^{5}$ The enhanced permeability of BRAF inhibitors by radiation can increase radiosensitivity and may improve local control. When BRAF inhibitor therapy was given before SRS, we did not find an association with local control; however, this may solely be a result of the relatively small number of patients who received BRAF inhibitor therapy in that setting. When we added patients who also received BRAF inhibitor therapy in the adjuvant setting, we noted improved local control.

Although BRAF inhibitor therapy is associated with improved local control, it is not associated with improved distant brain control. Despite significant CNS response rates, vemurafenib and dabrafenib do not cross the bloodbrain barrier in high concentrations. ${ }^{27,28}$ The median time to distant brain progression in our cohort of patients was 5.5 months, which compares to the 4.4-month median duration of intracranial response noted by Dummer et al. ${ }^{9}$ in patients who received vemurafenib. However, in these previous studies in which the role of BRAF inhibitors was examined, distant brain control was not specifically studied. ${ }^{9,25}$ Because SRS is a targeted treatment, it can likely focally improve the blood-brain barrier penetration of a BRAF inhibitor. We hypothesize that BRAF inhibitors failed to impact distant brain control because the bloodbrain barrier remains intact at other sites not treated with SRS. In addition, acquired resistance from BRAF therapy may affect distant brain control. ${ }^{10}$ Overcoming resistance may necessitate focusing on molecular targets, such as MEK. ${ }^{15}$ The use of combined BRAF and MEK inhibitors has resulted in improved progression-free survival. ${ }^{12}$ This study underscores the importance of developing therapies that can overcome the blood-brain barrier or be delivered intrathecally.

Chapman et al. ${ }^{7}$ previously showed an increase in the overall survival rate from $64 \%$ in the dacarbazine cohort to $84 \%$ with vemurafenib at 6 months in patients with metastatic melanoma. In our study, patients who were treated with BRAF inhibitor therapy did not experience an improved overall survival. Patients with brain metastases were enrolled onto the study only if those brain metastases were treated definitively more than 3 months before
TABLE 3. Univariate analysis of local control

\begin{tabular}{lcc}
\hline \multicolumn{1}{c}{ Variable } & $\mathrm{HR}(95 \% \mathrm{Cl})$ & $\mathrm{p} \mathrm{Value}$ \\
\hline Age & $1.00(0.97-1.03)$ & 1.00 \\
\hline Male sex & $0.61(0.27-1.34)$ & 0.14 \\
\hline Treatment volume & $1.00(0.92-1.08)$ & 0.95 \\
\hline BRAF mutation status & $1.54(0.75-3.14)$ & 0.19 \\
\hline Radiation dose & $1.00(1.00-1.001)$ & 0.91 \\
\hline Surgery before SRS & $0.714(0.21-2.34)$ & 0.52 \\
\hline
\end{tabular}

enrollment and had no progression. ${ }^{7}$ It was not noted how many of the patients had distant metastatic brain disease. ${ }^{7}$ Our population was different, because our patients had known brain metastases that were treated with SRS.

In our cohort, we determined that a new hemorrhage occurred in 50 lesions $(29.2 \%)$. Hemorrhage was associated with BRAF inhibitor therapy in our study; the rate of freedom from intratumoral hemorrhage was higher in patients who did not have BRAF therapy (77\%) than in those who did have BRAF therapy (39\%). SRS is not a risk factor for subsequent hemorrhage; a previous study by Ghia et al. ${ }^{14}$ found no association between SRS and the risk of subsequent hemorrhage in patients with melanoma brain metastases. Our report of increased risk of hemorrhage from BRAF therapy contradicts previous reports. Twentyfour patients with melanoma brain metastases who were treated with vemurafenib experienced mostly mild to moderate adverse effects, and no intracranial adverse effects were noted. ${ }^{9}$ In a study by Long et al. ${ }^{25} 172$ patients received dabrafenib for metastatic brain lesions, and 10 (6\%) had an intracranial hemorrhage, although only 1 was attributed to treatment. BRAF therapy with SRS in our cohort was associated with intratumoral hemorrhage.

Intratumoral hemorrhage may be a risk for death; a review of 333 patients with melanoma who were treated with SRS alone for brain metastases showed that $21 \%$ of them developed intratumoral hemorrhage. Hemorrhage was associated with decreased patient survival. ${ }^{24}$ In our cohort, 15 (75\%) deaths of CNS etiology were related to intratumoral hemorrhage of a previously treated tumor. Overall survival was not different between our BRAF therapy groups. Despite the improved local control with BRAF inhibitor therapy, we hypothesize that overall survival was not different because of the higher rates of hemorrhage that were associated with BRAF inhibitor therapy. It is not certain whether there is a causative relationship between BRAF inhibitor therapy and hemorrhage in patients who received SRS. Previous trials in which the use of BRAF inhibitor therapy in the intracranial setting was examined did not have comparison groups to assess the differences in hemorrhage. ${ }^{9,25}$ In a series of 5 patients who received whole-brain radiation or SRS along with BRAF inhibitor therapy, there was no evidence of increased intracranial toxicity, including hemorrhage. ${ }^{33}$ Hemorrhage may not be the only intracranial toxicity. In a recent case report, 2 patients developed radiation necrosis of the brain after being treated with SRS followed by vemurafenib 1-2 weeks later. ${ }^{23}$ In a separate report, a patient developed radiation necrosis of the brain after receiving SRS while 
on vemurafenib. ${ }^{31}$ In our series, all the patients underwent SRS, which may have potentiated the effect of BRAF inhibitors and its toxicity.

Increased toxicity, including hemorrhage, has been noted in other organ sites with BRAF inhibitor therapy both with and without radiation therapy. In one case report, a patient with melanoma developed a splenic hematoma and capsule laceration without evidence of metastases after vemurafenib therapy. ${ }^{6}$ In another case report, Trappe et al. ${ }^{40}$ noted a patient with melanoma who developed disseminated intravascular coagulation after vemurafenib therapy. It was speculated that rapid regression of melanoma may have led to disseminated intravascular coagulation and proteolysis. ${ }^{40}$ In a case report by Anker et al., ${ }^{1}$ a patient with melanoma who was treated with vemurafenib experienced in-field hepatic injury with fatal hepatic hemorrhage after palliative spine radiation. Others have reported varying skin, ${ }^{1-3,13,31,36,41}$ lung, and bowel toxicities in patients after treatment with both radiation and BRAF inhibitors. The degree and length of the duration of these toxicities are concerning. ${ }^{31}$ Because of these reports and our findings, future studies should examine the relationship of BRAF inhibitor therapy and the use of radiation therapy in the intracranial setting. We recommend discontinuation of BRAF inhibitors for 1-2 weeks both before and after SRS treatment, provided systemic disease is controlled. We await data from a recently completed Phase II study of vemurafenib (ClinicalTrials.gov identifier NCT01378975), in which the efficacy and safety of treatment in patients with metastatic melanoma with brain metastases were assessed.

Our study is limited in that it was a retrospective review of our treated patients. BRAF inhibitor therapy was not initiated in a prospective manner but was typically given after failure of previous systemic agents. In our study, BRAF inhibitor therapy could have been given before and/or after SRS after a washout period. Because this was a retrospective study, the initiation of BRAF inhibitor therapy may have been biased and given to healthier patients. However, comparisons of demographics including age, Karnofsky Performance Scale scores, and recursive partitioning analysis classifications did not reveal significant associations between the BRAF-inhibitor-treatment group and the noBRAF-inhibitor-treatment group. Another limitation of this study is that the initiation of BRAF therapy could have been given before or after SRS. When we evaluated the use of BRAF therapy when given only before SRS, there was no significant association; however, this may have been a result of the lack of patients in that cohort. The use of BRAF inhibitor therapy along with radiation should warrant a formal clinical trial based on the likelihood of radiosensitization with BRAF inhibitor therapies along with our finding of an association with improved local control with BRAF inhibitor therapy. Future clinical trials should randomly assign patients with $B R A F$ mutation who have melanoma and brain metastases to receive SRS with BRAF inhibitor therapy or SRS alone.

\section{Conclusions}

To our knowledge, this is the first study to show an association for improved local control with the addition of
BRAF inhibitors in patients with melanoma brain metastases treated with SRS. The addition of BRAF inhibitor therapy to SRS in melanoma brain metastases was found to be associated with an increased risk of intratumoral hemorrhage. Because of the potential toxicity of BRAF inhibitors, we recommend discontinuation for 1-2 weeks before and after SRS treatment, provided systemic disease is controlled.

\section{References}

1. Anker CJ, Ribas A, Grossmann AH, Chen X, Narra KK, Akerley W, et al: Severe liver and skin toxicity after radiation and vemurafenib in metastatic melanoma. J Clin Oncol 31:e283-e287, 2013

2. Boussemart L, Boivin C, Claveau J, Tao YG, Tomasic G, Routier E, et al: Vemurafenib and radiosensitization. JAMA Dermatol 149:855-857, 2013

3. Braunstein I, Gangadhar TC, Elenitsas R, Chu EY: Vemurafenib-induced interface dermatitis manifesting as radiation-recall and a keratosis pilaris-like eruption. J Cutan Pathol 41:539-543, 2014

4. Brown PD, Brown CA, Pollock BE, Gorman DA, Foote RL: Stereotactic radiosurgery for patients with "radioresistant" brain metastases. Neurosurgery 62 (Suppl 2):790-801, 2008

5. Cao Y, Tsien CI, Shen Z, Tatro DS, Ten Haken R, Kessler $\mathrm{ML}$, et al: Use of magnetic resonance imaging to assess blood-brain/blood-glioma barrier opening during conformal radiotherapy. J Clin Oncol 23:4127-4136, 2005

6. Castellani E, Covarelli P, Boselli C, Cirocchi R, Rulli A, Barberini F, et al: Spontaneous splenic rupture in patient with metastatic melanoma treated with vemurafenib. World J Surg Oncol 10:155, 2012

7. Chapman PB, Hauschild A, Robert C, Haanen JB, Ascierto P, Larkin J, et al: Improved survival with vemurafenib in melanoma with BRAF V600E mutation. N Engl J Med 364:2507-2516, 2011

8. Davies H, Bignell GR, Cox C, Stephens P, Edkins S, Clegg S, et al: Mutations of the BRAF gene in human cancer. Nature 417:949-954, 2002

9. Dummer R, Goldinger SM, Turtschi CP, Eggmann NB, Michielin O, Mitchell L, et al: Vemurafenib in patients with BRAF(V600) mutation-positive melanoma with symptomatic brain metastases: final results of an open-label pilot study. Eur J Cancer 50:611-621, 2014

10. Emery CM, Vijayendran KG, Zipser MC, Sawyer AM, Niu L, Kim JJ, et al: MEK1 mutations confer resistance to MEK and B-RAF inhibition. Proc Natl Acad Sci U S A 106:2041120416, 2009

11. Falchook GS, Long GV, Kurzrock R, Kim KB, Arkenau TH, Brown MP, et al: Dabrafenib in patients with melanoma, untreated brain metastases, and other solid tumours: a phase 1 dose-escalation trial. Lancet 379:1893-1901, 2012

12. Flaherty KT, Infante JR, Daud A, Gonzalez R, Kefford RF, Sosman J, et al: Combined BRAF and MEK inhibition in melanoma with BRAF V600 mutations. N Engl J Med 367:1694-1703, 2012

13. Forschner A, Niessner H, Bauer J, Bender B, Garbe C, Meier F: Successful treatment with vemurafenib in BRAF V600Kpositive cerebral melanoma metastasis. JAMA Dermatol 149:642-644, 2013

14. Ghia AJ, Tward JD, Anker CJ, Boucher KM, Jensen RL, Shrieve DC: Radiosurgery for melanoma brain metastases: the impact of hemorrhage on local control. J Radiosurg SBRT 3:43-50, 2014

15. Greger JG, Eastman SD, Zhang V, Bleam MR, Hughes AM, Smitheman KN, et al: Combinations of BRAF, MEK, and $\mathrm{PI} 3 \mathrm{~K} / \mathrm{mTOR}$ inhibitors overcome acquired resistance to the BRAF inhibitor GSK2118436 dabrafenib, mediated by 
NRAS or MEK mutations. Mol Cancer Ther 11:909-920, 2012

16. Gumusay O, Coskun U, Akman T, Ekinci AS, Kocar M, Erceleb OB, et al: Predictive factors for the development of brain metastases in patients with malignant melanoma: a study by the Anatolian society of medical oncology. J Cancer Res Clin Oncol 140:151-157, 2014

17. Hauschild A, Grob JJ, Demidov LV, Jouary T, Gutzmer R, Millward M, et al: Dabrafenib in BRAF-mutated metastatic melanoma: a multicentre, open-label, phase 3 randomised controlled trial. Lancet 380:358-365, 2012

18. Hazard LJ, Wang B, Skidmore TB, Chern SS, Salter BJ, Jensen RL, et al: Conformity of LINAC-based stereotactic radiosurgery using dynamic conformal arcs and micro-multileaf collimator. Int J Radiat Oncol Biol Phys 73:562-570, 2009

19. Jahanshahi P, Nasr N, Unger K, Batouli A, Gagnon GJ: Malignant melanoma and radiotherapy: past myths, excellent local control in 146 studied lesions at Georgetown University, and improving future management. Front Oncol 2:167, 2012

20. Jemal A, Saraiya M, Patel P, Cherala SS, Barnholtz-Sloan J, Kim J, et al: Recent trends in cutaneous melanoma incidence and death rates in the United States, 1992-2006. J Am Acad Dermatol 65:S17.e1-s17.e11, 2011

21. Jensen RL, Shrieve AF, Samlowski W, Shrieve DC: Outcomes of patients with brain metastases from melanoma and renal cell carcinoma after primary stereotactic radiosurgery. Clin Neurosurg 55:150-159, 2008

22. Karasarides M, Chiloeches A, Hayward R, Niculescu-Duvaz D, Scanlon I, Friedlos F, et al: B-RAF is a therapeutic target in melanoma. Oncogene 23:6292-6298, 2004

23. Liebner DA, Walston SA, Cavaliere R, Powers CJ, Sauvageau E, Lehman NL, et al: Radiation necrosis mimicking rapid intracranial progression of melanoma metastasis in two patients treated with vemurafenib. Melanoma Res 24:172-176, 2014

24. Liew DN, Kano H, Kondziolka D, Mathieu D, Niranjan A, Flickinger JC, et al: Outcome predictors of Gamma Knife surgery for melanoma brain metastases. Clinical article. J Neurosurg 114:769-779, 2011

25. Long GV, Trefzer U, Davies MA, Kefford RF, Ascierto PA, Chapman PB, et al: Dabrafenib in patients with Val600Glu or Val600Lys BRAF-mutant melanoma metastatic to the brain (BREAK-MB): a multicentre, open-label, phase 2 trial. Lancet Oncol 13:1087-1095, 2012

26. Lwu S, Goetz P, Monsalves E, Aryaee M, Ebinu J, Laperriere $\mathrm{N}$, et al: Stereotactic radiosurgery for the treatment of melanoma and renal cell carcinoma brain metastases. Oncol Rep 29:407-412, 2013

27. Mittapalli RK, Vaidhyanathan S, Dudek AZ, Elmquist WF: Mechanisms limiting distribution of the threonine-protein kinase $\mathrm{B}-\mathrm{RaF}(\mathrm{V} 600 \mathrm{E})$ inhibitor dabrafenib to the brain: implications for the treatment of melanoma brain metastases. J Pharmacol Exp Ther 344:655-664, 2013

28. Mittapalli RK, Vaidhyanathan S, Sane R, Elmquist WF: Impact of P-glycoprotein (ABCB1) and breast cancer resistance protein (ABCG2) on the brain distribution of a novel BRAF inhibitor: vemurafenib (PLX4032). J Pharmacol Exp Ther 342:33-40, 2012

29. Narayana A, Mathew M, Tam M, Kannan R, Madden KM, Golfinos JG, et al: Vemurafenib and radiation therapy in melanoma brain metastases. J Neurooncol 113:411-416, 2013

30. Neal MT, Chan MD, Lucas JT Jr, Loganathan A, Dillingham C, Pan E, et al: Predictors of survival, neurologic death, local failure, and distant failure after gamma knife radiosurgery for melanoma brain metastases. World Neurosurg 82:12501255,2014

31. Peuvrel L, Ruellan AL, Thillays F, Quereux G, Brocard A, Saint-Jean M, et al: Severe radiotherapy-induced extracutaneous toxicity under vemurafenib. Eur J Dermatol 23:879_ 881,2013
32. Powell JW, Chung CT, Shah HR, Canute GW, Hodge CJ, Bassano DA, et al: Gamma Knife surgery in the management of radioresistant brain metastases in high-risk patients with melanoma, renal cell carcinoma, and sarcoma. J Neurosurg 109 Suppl:122-128, 2008

33. Rompoti N, Schilling B, Livingstone E, Griewank K, Hillen U, Sauerwein W, et al: Combination of BRAF inhibitors and brain radiotherapy in patients with metastatic melanoma shows minimal acute toxicity. J Clin Oncol 31:3844-3845, 2013

34. Sambade MJ, Peters EC, Thomas NE, Kaufmann WK, Kimple RJ, Shields JM: Melanoma cells show a heterogeneous range of sensitivity to ionizing radiation and are radiosensitized by inhibition of B-RAF with PLX-4032. Radiother Oncol 98:394-399, 2011

35. Sampson JH, Carter JH Jr, Friedman AH, Seigler HF: Demographics, prognosis, and therapy in 702 patients with brain metastases from malignant melanoma. J Neurosurg 88:11-20, 1998

36. Satzger I, Degen A, Asper H, Kapp A, Hauschild A, Gutzmer R: Serious skin toxicity with the combination of BRAF inhibitors and radiotherapy. J Clin Oncol 31:e220-e222, 2013

37. Schouten LJ, Rutten J, Huveneers HA, Twijnstra A: Incidence of brain metastases in a cohort of patients with carcinoma of the breast, colon, kidney, and lung and melanoma. Cancer 94:2698-2705, 2002

38. Selek U, Chang EL, Hassenbusch SJ III, Shiu AS, Lang FF, Allen P, et al: Stereotactic radiosurgical treatment in 103 patients for 153 cerebral melanoma metastases. Int J Radiat Oncol Biol Phys 59:1097-1106, 2004

39. Simeone E, De Maio E, Sandomenico F, Fulciniti F, Lastoria $\mathrm{S}$, Aprea P, et al: Neoplastic leptomeningitis presenting in a melanoma patient treated with dabrafenib (a V600EBRAF inhibitor): a case report. J Med Case Reports 6:131, 2012

40. Trappe R, Budde U, Zimmermann H: Haemorrhage due to proteolysis of FXIII and hyperfibrinolysis under vemurafenib treatment of metastatic melanoma. Thromb Haemost 108:798-800, 2012

41. Wang CM, Fleming KF, Hsu S: A case of vemurafenibinduced keratosis pilaris-like eruption. Dermatol Online J 18:7, 2012

42. Zakrzewski J, Geraghty LN, Rose AE, Christos PJ, Mazumdar M, Polsky D, et al: Clinical variables and primary tumor characteristics predictive of the development of melanoma brain metastases and post-brain metastases survival. Cancer 117:1711-1720, 2011

\section{Author Contributions}

Conception and design: Shrieve, Ly. Acquisition of data: Ly, Bagshaw. Analysis and interpretation of data: all authors. Drafting the article: Shrieve, Ly. Critically revising the article: all authors. Reviewed submitted version of manuscript: all authors. Approved the final version of the manuscript on behalf of all authors: Shrieve. Statistical analysis: Shrieve, Ly, Bagshaw, Anker, Jensen. Administrative/technical/material support: Tward, Grossmann. Study supervision: Shrieve, Anker, Tward, Grossmann, Jensen.

\section{Supplemental Information}

Previous Presentation

Portions of this work were presented in poster form at the American Society for Radiation Oncology 55th Annual Meeting held in Atlanta, Georgia, on September 22-25, 2013.

\section{Correspondence}

Dennis C. Shrieve, Department of Radiation Oncology, University of Utah, Huntsman Cancer Hospital, 1950 Circle of Hope Dr., Ste. 1570, Salt Lake City, UT 84112. email: dennis. shrieve@hci.utah.edu. 